\title{
PERMANENT V. DETERMINANT: AN EXPONENTIAL LOWER BOUND ASSUMING SYMMETRY AND A POTENTIAL PATH TOWARDS VALIANT'S CONJECTURE
}

\author{
J.M. LANDSBERG AND NICOLAS RESSAYRE
}

\begin{abstract}
We initiate a study of determinantal representations with symmetry. We show that Grenet's determinantal representation for the permanent is optimal among determinantal representations respecting left multiplication by permutation and diagonal matrices (roughly half the symmetry group of the permanent). In particular, if any optimal determinantal representation of the permanent must be polynomially related to one with such symmetry, then Valiant's conjecture on permanent $\mathrm{v}$. determinant is true.
\end{abstract}

\section{INTRODUCTION}

Perhaps the most studied polynomial of all is the determinant:

$$
\operatorname{det}_{n}(x):=\sum_{\sigma \in \mathfrak{S}_{n}} \operatorname{sgn}(\sigma) x_{\sigma(1)}^{1} x_{\sigma(2)}^{2} \cdots x_{\sigma(n)}^{n},
$$

a homogeneous polynomial of degree $n$ in $n^{2}$ variables. Here $\mathfrak{S}_{n}$ denotes the group of permutations on $n$ elements and $\operatorname{sgn}(\sigma)$ denotes the sign of the permutation $\sigma$.

Despite its formula with $n$ ! terms, $\operatorname{det}_{n}$ can be evaluated quickly, e.g., using Gaussian elimination, which exploits the large symmetry group of the determinant, e.g., $\operatorname{det}_{n}(x)=\operatorname{det}_{n}\left(A x B^{-1}\right)$ for any $n \times n$ matrices $A, B$ with determinant equal to one.

We will work exclusively over the complex numbers and with homogeneous polynomials, the latter restriction only for convenience. L. Valiant showed that given a homogeneous polynomial $P(y)$ in $M$ variables, there exists an $n$ and an affine linear map $\tilde{A}: \mathbb{C}^{M} \rightarrow \mathbb{C}^{n^{2}}$ such that $P=\operatorname{det}_{n} \circ \tilde{A}$. Such $\tilde{A}$ is called a determinantal representation of $P$. When $M=m^{2}$ and $P$ is the permanent polynomial

$$
\operatorname{perm}_{m}(y):=\sum_{\sigma \in \mathfrak{S}_{m}} y_{\sigma(1)}^{1} y_{\sigma(2)}^{2} \cdots y_{\sigma(m)}^{m},
$$

L. Valiant showed that one can take $n=O\left(2^{m}\right)$. As an algebraic analog of the $\mathbf{P} \neq \mathbf{N P}$ conjecture, he also conjectured that one cannot do much better:

Key words and phrases. Geometric Complexity Theory, determinant, permanent, MSC 68Q15 (20G05).

Landsberg supported by NSF grant DMS-1405348. Ressayre supported by ANR Project (ANR-13-BS02-0001-01) and by Institut Universitaire de France. 
Conjecture 1.1 (Valiant [Val79]). Let $n(m)$ be a function of $m$ such that there exist affine linear maps $\tilde{A}_{m}: \mathbb{C}^{m^{2}} \rightarrow \mathbb{C}^{n(m)^{2}}$ satisfying

$$
\operatorname{perm}_{m}=\operatorname{det}_{n(m)} \circ \tilde{A}_{m} .
$$

Then $n(m)$ grows faster than any polynomial.

To measure progress towards Conjecture 1.1, define $\operatorname{dc}\left(\operatorname{perm}_{m}\right)$ to be the smallest $n(m)$ such that there exists $\tilde{A}_{m}$ satisfying (3). The conjecture is that $\operatorname{dc}\left(\operatorname{perm}_{m}\right)$ grows faster than any polynomial in $m$. Lower bounds on $\operatorname{dc}\left(\operatorname{perm}_{m}\right)$ are: $\operatorname{dc}\left(\operatorname{perm}_{m}\right)>m$ (Marcus and Minc [MM61]), $\operatorname{dc}\left(\operatorname{perm}_{m}\right)>1.06 m\left(\right.$ Von zur Gathen [vzG87]), $\operatorname{dc}\left(\operatorname{perm}_{m}\right)>\sqrt{2} m-O(\sqrt{m})$ (Meshulam, reported in [vzG87], and Cai [Cai90]), with the current world record $\mathrm{dc}\left(\right.$ perm $\left._{m}\right) \geq \frac{m^{2}}{2}$ [MR04] by Mignon and the second author. (Over $\mathbb{R}$, Yabe recently showed that $\operatorname{dc}_{\mathbb{R}}\left(\operatorname{perm}_{m}\right) \geq m^{2}-2 m+2$ [Yab15], and in [CCL10] Cai, Chen and Li extended the $\frac{m^{2}}{2}$ bound to arbitrary fields.)

Inspired by Geometric Complexity Theory (GCT) [MS01], we focus on the symmetries of $\operatorname{det}_{n}$ and $\operatorname{perm}_{m}$. Let $V$ be a complex vector space of dimension $M$, let GL( $V)$ denote the group of invertible linear maps $V \rightarrow V$. For $P \in S^{m} V^{*}$, a homogeneous polynomial of degree $m$ on $V$, let

$$
\begin{aligned}
& G_{P}:=\left\{g \in \mathrm{GL}(V) \mid P\left(g^{-1} y\right)=P(y) \quad \forall y \in V\right\} \\
& \mathbb{G}_{P}:=\left\{g \in \mathrm{GL}(V) \mid P\left(g^{-1} y\right) \in \mathbb{C}^{*} P(y) \quad \forall y \in V\right\}
\end{aligned}
$$

denote the symmetry group (resp. projective symmetry group) of $P$. The function $\chi_{P}: \mathbb{G}_{P} \rightarrow \mathbb{C}^{*}$ defined by the equality $P\left(g^{-1} y\right)=\chi_{P}(g) P(y)$ is group homomorphism called the character of $P$. For example $\mathbb{G}_{\operatorname{det}_{n}} \simeq$ $\left(\mathrm{GL}_{n} \times \mathrm{GL}_{n}\right) / \mathbb{C}^{\star} \rtimes \mathbb{Z}_{2}$ [Fro97], where the $\mathrm{GL}_{n} \times \mathrm{GL}_{n}$ invariance comes from

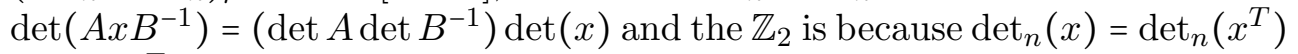
where $x^{T}$ is the transpose of the matrix $x$. Write $\tau: \mathrm{GL}_{n} \times \mathrm{GL}_{n} \rightarrow \mathrm{GL}_{n^{2}}$ for the map $(A, B) \mapsto\left\{x \mapsto A x B^{-1}\right\}$. The character $\chi_{\operatorname{det}_{n}}$ satisfies $\chi_{\operatorname{det}_{n}} \circ$ $\tau(A, B)=\operatorname{det}(A) \operatorname{det}(B)^{-1}$.

As observed in [MS01], the permanent (resp. determinant) is characterized by its symmetries in the sense that any polynomial $P \in S^{m} \mathbb{C}^{m^{2} *}$ with a symmetry group $G_{P}$ such that $G_{P} \supseteq G_{\text {perm }_{m}}$ (resp. $G_{P} \supseteq G_{\operatorname{det}_{m}}$ ) is a scalar multiple of the permanent (resp. determinant). This property is the cornerstone of GCT. The program outlined in [MS01, MS08] is an approach to Valiant's conjecture based on the functions on $\mathrm{GL}_{n^{2}}$ that respect the symmetry group $G_{\operatorname{det}_{n}}$, i.e., are invariant under the action of $G_{\operatorname{det}_{n}}$.

The interest in considering $\mathbb{G}_{P}$ instead of $G_{P}$ is that if $P$ is characterized by $G_{P}$ among homogeneous polynomials of the same degree, then it is characterized by the pair $\left(\mathbb{G}_{P}, \chi_{P}\right)$ among all polynomials. This will be useful, since a priori, $\operatorname{det}_{n} \circ \tilde{A}$ need not be homogeneous.

Guided by the principles of GCT, we ask:

What are the $\tilde{A}$ that respect the symmetry group of the permanent?

To make this question precise, let $\mathcal{M}_{n}(\mathbb{C})$ denote the space of $n \times n$ matrices and write $\tilde{A}=\Lambda+A$ where $\Lambda \in \mathcal{M}_{n}(\mathbb{C})$ is a fixed matrix and $A: \mathcal{M}_{m}(\mathbb{C}) \rightarrow \mathcal{M}_{n}(\mathbb{C})$ is linear. 
The subgroup $\mathbb{G}_{\operatorname{det}_{n}} \subset \mathrm{GL}_{n^{2}}$ satisfies

$$
\mathbb{G}_{\operatorname{det}_{n}} \simeq\left(\mathrm{GL}_{n} \times \mathrm{GL}_{n}\right) / \mathbb{C}^{*} \rtimes \mathbb{Z}_{2} .
$$

Definition 1.2. Let $\tilde{A}: V \longrightarrow \mathcal{M}_{n}(\mathbb{C})$ be a determinantal representation of $P \in S^{m} V^{*}$. Define

$$
\mathbb{G}_{A}=\left\{g \in \mathbb{G}_{\operatorname{det}_{n}} \mid g \cdot \Lambda=\Lambda \text { and } g \cdot A(V)=A(V)\right\},
$$

the symmetry group of the determinantal representation $\tilde{A}$ of $P$.

The group $\mathbb{G}_{A}$ comes with a representation $\rho_{A}: \mathbb{G}_{A} \longrightarrow \mathrm{GL}(A(V))$ obtained by restricting the action to $A(V)$. We assume that $P$ cannot be expressed using $\operatorname{dim}(V)-1$ variables, i.e., that $P \notin S^{m} V^{\prime}$ for any hyperplane $V^{\prime} \subset V^{*}$. Then $A: V \longrightarrow A(V)$ is bijective. Let $A^{-1}: A(V) \longrightarrow V$ denote its inverse. Set

$$
\begin{aligned}
\bar{\rho}_{A}: \mathbb{G}_{A} & \longrightarrow \mathrm{GL}(V) \\
g & \longmapsto A \circ \rho_{A}(g) \circ A^{-1} .
\end{aligned}
$$

Definition 1.3. We say $\tilde{A}$ respects the symmetries of $P$ if (5) is surjective. We also refer to such $\tilde{A}$ as a equivariant representation of $P$.

If $G$ is a subgroup of $\mathbb{G}_{P}$, we say that $\tilde{A}$ respects $G$ if $G$ is contained in the image of $\bar{\rho}_{A}$.

Example 1.4. Let $Q=\sum_{j=1}^{M} z_{j}^{2} \in S^{2} \mathbb{C}^{M *}$ be a nondegenerate quadric. Then $\mathbb{G}_{Q}=\mathbb{C}^{*} \times O(M)$ where $O(M)=\left\{B \in G L_{M} \mid B^{-1}=B^{T}\right\}$ is the orthogonal group, as for such $B, B \cdot Q=\sum_{i, j, k} B_{i, j} B_{k, j} z_{i} z_{k}=\sum_{i j} \delta_{i j} z_{i} z_{j}=Q$. Consider the determinantal representation

$$
Q=\operatorname{det}_{M+1}\left(\begin{array}{cccc}
0 & -z_{1} & \cdots & -z_{M} \\
z_{1} & 1 & & \\
\vdots & & \ddots & \\
z_{M} & & & 1
\end{array}\right)
$$

For $(\lambda, B) \in \mathbb{G}_{Q}$, define an action on $Z \in \mathcal{M}_{M+1}(\mathbb{C})$ by

$$
Z \mapsto\left(\begin{array}{cc}
\lambda & 0 \\
0 & B
\end{array}\right) Z\left(\begin{array}{cc}
\lambda^{-1} & 0 \\
0 & B
\end{array}\right)^{-1}
$$

Write

$$
X=\left(\begin{array}{c}
x_{1} \\
\vdots \\
x_{M}
\end{array}\right) \quad \text { so } \quad \tilde{A}=\left(\begin{array}{cc}
0 & -X^{T} \\
X & \mathrm{Id}_{M}
\end{array}\right)
$$

The relation $B^{-1}=B^{T}$ implies

$$
\left(\begin{array}{cc}
\lambda & 0 \\
0 & B
\end{array}\right) \cdot\left(\begin{array}{cc}
0 & -X^{T} \\
X & \operatorname{Id}_{M}
\end{array}\right) \cdot\left(\begin{array}{cc}
\lambda^{-1} & 0 \\
0 & B
\end{array}\right)^{-1}=\left(\begin{array}{cc}
0 & -(\lambda B X)^{T} \\
\lambda B X & \operatorname{Id}_{M}
\end{array}\right)
$$

Taking $\operatorname{det}_{M+1}$ on both sides gives

$$
\lambda^{2} Q(X)=(\lambda, B) \cdot Q(X) .
$$

Thus $\tilde{A}$ respects the symmetries of $Q$. 
Roughly speaking, $\tilde{A}$ respects the symmetries of $P$ if for any $g \in \mathbb{G}_{P}$, one can recover the fact that $P\left(g^{-1} x\right)=\chi_{P}(g) P(x)$ just by applying Gaussian elimination and $\operatorname{det}\left(Z^{T}\right)=\operatorname{det}(Z)$ to $\tilde{A}$.

Definition 1.5. For $P \in S^{m} V^{*}$, define the equivariant determinantal complexity of $P$, denoted edc $(P)$, to be the smallest $n$ such that there is an equivariant determinantal representation of $P$.

Of course edc $(P) \geq \operatorname{dc}(P)$. We do not know if edc $(P)$ is finite in general. Our main result is that edc $\left(\operatorname{perm}_{m}\right)$ is exponential in $m$.

\section{Results}

\subsection{Main Theorem.}

Theorem 2.1. Let $m \geq 3$. Then edc $\left(\operatorname{perm}_{m}\right)=\left(\begin{array}{c}2 m \\ m\end{array}\right)-1 \sim 4^{m}$.

There are several instances in complexity theory where an optimal algorithm partially respects symmetry, e.g. Strassen's algorithm for $2 \times 2$ matrix multiplication respects the $\mathbb{Z}_{3}$-symmetry of the matrix multiplication operator (see [Lan, §4.2]), but not the $G L_{2}^{\times 3}$ symmetry.

For the purposes of Valiant's conjecture, we ask the weaker question:

Question 2.2. Does there exist a polynomial $e(d) \operatorname{such}$ that edc $\left(\operatorname{perm}_{m}\right) \leq$ $e\left(\operatorname{dc}\left(\operatorname{perm}_{m}\right)\right)$ ?

Theorem 2.1 implies:

Corollary 2.3. If the answer to Question 2.2 is affirmative, then Conjecture 1.1 is true.

We have no opinion as to what the answer to Question 2.2 should be, but as it provides a new potential path to proving Valiant's conjecture, it merits further investigation. Note that Question 2.2 is a flip in the terminology of [Mul], since a positive answer is an existence result. It fits into the more general question: When an object has symmetry, does it admit an optimal expression that preserves its symmetry?

Example 2.4. Let $T \in W^{\otimes d}$ be a symmetric tensor, i.e. $T \in S^{d} W \subset W^{\otimes d}$. Say $T$ can be written as a sum of $r$ rank one tensors, then P. Comon conjectures [Com02] that it can be written as a sum of $r$ rank one symmetric tensors.

Example 2.5 (Optimal Waring decompositions). The optimal Waring decomposition of $x_{1} \ldots x_{n}$, dating back at least to [Fis94] and proved to be optimal in [RS11] is

$$
x_{1} \ldots x_{n}=\frac{1}{2^{n-1} n !} \sum_{\substack{\epsilon \in\{-1,1\}^{n} \\ \epsilon_{1}=1}}\left(\sum_{j=1}^{n} \epsilon_{j} x_{j}\right)^{n} \Pi_{i=1}^{n} \epsilon_{i},
$$

a sum with $2^{n-1}$ terms. This decomposition has an $\mathfrak{S}_{n-1}$-symmetry but not an $\mathfrak{S}_{n}$-symmetry, nor does it preserve the action of the torus $T^{S L_{n}}$ of diagonal matrices with determinant one. One can obtain an $\mathfrak{S}_{n}$-invariant expression by doubling the size:

$$
x_{1} \ldots x_{n}=\frac{1}{2^{n} n !} \sum_{\epsilon \in\{-1,1\}^{n}}\left(\sum_{j=1}^{n} \epsilon_{j} x_{j}\right)^{n} \Pi_{i=1}^{n} \epsilon_{i},
$$


because

$$
\begin{aligned}
\left(-x_{1}+\epsilon_{2} x_{2}+\cdots\right. & \left.+\epsilon_{n} x_{n}\right)^{n}(-1) \epsilon_{2} \ldots \epsilon_{n} \\
& =(-1)^{n}\left(x_{1}+\left(-\epsilon_{2}\right) x_{2}+\cdots+\left(-\epsilon_{n}\right) x_{n}\right)^{n}(-1) \epsilon_{2} \ldots \epsilon_{n} \\
& =\left(x_{1}+\left(-\epsilon_{2}\right) x_{2}+\cdots+\left(-\epsilon_{n}\right) x_{n}\right)^{n}\left(-\epsilon_{2}\right) \ldots\left(-\epsilon_{n}\right) .
\end{aligned}
$$

The optimal Waring decomposition of the permanent is not known, but it is known to be of size greater than $\left(\begin{array}{c}n \\ \lfloor n / 2\rfloor\end{array}\right)^{2} \sim 4^{n} / \sqrt{n}$. The Ryser-Glynn formula [Gly10] is

$$
\operatorname{perm}_{n}(x)=2^{-n+1} \sum_{\substack{\epsilon \in\{-1,1\}^{n} \\ \epsilon_{1}=1}} \prod_{1 \leq i \leq n} \sum_{1 \leq j \leq n} \epsilon_{i} \epsilon_{j} x_{i, j}
$$

the outer sum taken over $n$-tuples $\left(\epsilon_{1}=1, \epsilon_{2}, \ldots, \epsilon_{n}\right)$. This $\mathfrak{S}_{n-1} \times \mathfrak{S}_{n-1^{-}}$ invariant formula can also be made $\mathfrak{S}_{n} \times \mathfrak{S}_{n}$-invariant by enlarging it by a factor of 4 , to get a $\mathfrak{S}_{n} \times \mathfrak{S}_{n}$ homogeneous depth three formula that is within a factor of four of the best known. Then expanding each monomial above, using Equation (8), one gets a $\mathfrak{S}_{n} \times \mathfrak{S}_{n}$-Waring expression within a factor of $O(\sqrt{n})$ of the lower bound.

Example 2.6. Examples regarding equivariant representations of $\mathfrak{S}_{N}$-invariant functions from the Boolean world give inconclusive indications regarding Question 2.2.

The $M O D_{m}$-degree of a Boolean function $f\left(x_{1}, \cdots, x_{N}\right)$ is the smallest degree of any polynomial $P \in \mathbb{Z}\left[x_{1}, \cdots, x_{N}\right]$ such that $f(x)=0$ if and only if $P(x)=0$ for all $x \in\{0,1\}^{N}$. The known upper bound for the $M O D_{m}$-degree of the Boolean $O R$ function $\operatorname{OR}\left(x_{1}, \cdots, x_{N}\right)=1$ if any $x_{j}=1$ and is zero if all $\left.x_{j}=0\right)$ is attained by symmetric polynomials [BBR94]. Moreover in [BBR94] it is also shown that this bound cannot be improved with symmetric polynomials, and it is far from the known lower bound.

The boolean majority function $M A J\left(x_{1}, \cdots, x_{N}\right)$ takes on 1 if at least half the $x_{j}=1$ and zero otherwise. The best monotone Boolean formula for $M A J$ [Val84] is polynomial in $N$ and attained using random functions, and it is expected that the only symmetric monotone formula for majority is the trivial one, disjunction of all $\frac{n}{2}$-size subsets (or its dual), which is of exponential size.

Question 2.7. Does every $P$ that is determined by its symmetry group admit a determinantal representation that respects its symmetries? For those $P$ that do, how much larger must such a determinantal representation be from the size of a minimal one? 
2.2. Grenet's formulas. The starting point of our investigations was the result in $[\mathrm{ABV} 15]$ that $\mathrm{dc}\left(\mathrm{perm}_{3}\right)=7$, in particular Grenet's representatation [Gre14] for perm 3 :

$$
\operatorname{perm}_{3}(y)=\operatorname{det}_{7}\left(\begin{array}{ccccccc}
0 & 0 & 0 & 0 & y_{3}^{3} & y_{2}^{3} & y_{1}^{3} \\
y_{1}^{1} & 1 & & & & & \\
y_{2}^{1} & & 1 & & & & \\
y_{3}^{1} & & & 1 & & & \\
& y_{2}^{2} & y_{1}^{2} & 0 & 1 & & \\
& y_{3}^{2} & 0 & y_{1}^{2} & & 1 & \\
& 0 & y_{3}^{2} & y_{2}^{2} & & & 1
\end{array}\right),
$$

is optimal. We sought to understand (10) from a geometric perspective. A first observation is that it, and more generally Grenet's representation for $\operatorname{perm}_{m}$ as a determinant of size $2^{m}-1$ respects about half the symmetries of the permanent. In particular, the optimal expression for perm ${ }_{3}$ respects about half its symmetries.

To explain this observation, introduce the following notation. Write $\mathcal{M}_{m}(\mathbb{C})=\operatorname{Hom}(F, E)=F^{*} \otimes E$, where $E, F=\mathbb{C}^{m}$. This distinction of the two copies of $\mathbb{C}^{m}$ clarifies the action of the group $\mathrm{GL}(E) \times \mathrm{GL}(F)$ on $\operatorname{Hom}(F, E)$. This action is $(A, B) \cdot x=A x B^{-1}$, for any $x \in \operatorname{Hom}(F, E)$ and $(A, B) \in \mathrm{GL}(E) \times \mathrm{GL}(F)$. Let $T^{\mathrm{GL}(E)} \subset \mathrm{GL}(E)$ consist of the diagonal matrices and let $N\left(T^{\mathrm{GL}(E)}\right)=T^{\mathrm{GL}(E)} \rtimes \mathfrak{S}_{m} \subset \mathrm{GL}(E)$ be its normalizer, where $\mathfrak{S}_{m}$ denotes the group of permutations on $m$ elements. Similarly for $T^{\mathrm{GL}(F)}$ and $N\left(T^{\mathrm{GL}(F)}\right)$. Then $\mathbb{G}_{\mathrm{perm}_{m}} \simeq\left[\left(N\left(T^{\mathrm{GL}(E)}\right) \times N\left(T^{\mathrm{GL}(F)}\right)\right) / \mathbb{C}^{*}\right] \rtimes \mathbb{Z}_{2}$, where the embedding of $\left(N\left(T^{\mathrm{GL}(E)}\right) \times N\left(T^{\mathrm{GL}(F)}\right)\right) / \mathbb{C}^{*}$ in $\mathrm{GL}(\operatorname{Hom}(F, E))$ is given by the action above and the term $\mathbb{Z}_{2}$ corresponds to transposition.

The following refinement of Theorem 2.1 asserts that to get an exponential lower bound it is sufficient to respect about half the symmetries of the permanent.

Theorem 2.8. Let $m \geq 3$. Let $\tilde{A}_{m}: \mathcal{M}_{m}(\mathbb{C}) \longrightarrow \mathcal{M}_{n}(\mathbb{C})$ be a determinantal representation of $\operatorname{perm}_{m}$ that respects $N\left(T^{\mathrm{GL}(E)}\right)$. Then $n \geq 2^{m}-1$.

Moreover, Grenet's determinantal representation of perm $_{m}$ respects $N\left(T^{\mathrm{GL}(E)}\right)$ and has size $2^{m}-1$.

We now explain Grenet's expressions from a representation-theoretic perspective. Let $[m]:=\{1, \cdots, m\}$ and let $k \in[m]$. Note that $S^{k} E$ is an irreducible $\mathrm{GL}(E)$-module but it is is not irreducible as an $N\left(T^{\mathrm{GL}(E)}\right)$-module. For example, let $e_{1}, \cdots, e_{m}$ be a basis of $E$, and let $\left(S^{k} E\right)_{\text {reg }}$ denote the span of $\prod_{i \in I} e_{i}$, for $I \subset[m]$ of cardinality $k$ (the space spanned by the square-free monomials, also known as the space of regular weights): $\left(S^{k} E\right)_{r e g}$ is an irreducible $N\left(T^{\mathrm{GL}(E)}\right)$-submodule of $S^{k} E$. Moreover, there exists a unique $N\left(T^{\mathrm{GL}(E)}\right)$-equivariant projection $\pi_{k}$ from $S^{k} E$ to $\left(S^{k} E\right)_{\text {reg }}$.

For $v \in E$, define $s_{k}(v):\left(S^{k} E\right)_{\text {reg }} \rightarrow\left(S^{k+1} E\right)_{\text {reg }}$ to be multiplication by $v$ followed by $\pi_{k+1}$. Alternatively, $\left(S^{k+1} E\right)_{\text {reg }}$ is an $N\left(T^{\mathrm{GL}(E)}\right)$-submodule of 
$E \otimes\left(S^{k} E\right)_{r e g}$, and $s_{k}: E \rightarrow\left(S^{k} E\right)_{r e g}^{*} \otimes\left(S^{k+1} E\right)_{r e g}$ is the unique $N\left(T^{\mathrm{GL}(E)}\right)$ equivariant inclusion. Let $\operatorname{Id}_{W}: W \rightarrow W$ denote the identity map on the vector space $W$. Fix a basis $f_{1}, \cdots, f_{m}$ of $F^{*}$.

Proposition 2.9. The following is Grenet's determinantal representation of perm $_{m}$. Let $\mathbb{C}^{n}=\bigoplus_{k=0}^{m-1}\left(S^{k} E\right)_{\text {reg }}$, so $n=2^{m}-1$, and identify $S^{0} E \simeq$ $\left(S^{m} E\right)_{\text {reg. }}$. Set

$$
\Lambda_{0}=\sum_{k=1}^{m-1} \operatorname{Id}_{\left(S^{k} E\right)_{r e g}}
$$

and define

$$
\tilde{A}=\Lambda_{0}+\sum_{k=0}^{m-1} s_{k} \otimes f_{k+1}
$$

Then $(-1)^{m+1} \operatorname{perm}_{m}=\operatorname{det}_{n} \circ \tilde{A}$. To obtain the permanent exactly, replace $\operatorname{Id}_{\left(S^{1} E\right)_{r e q}}$ by $(-1)^{m+1} \operatorname{Id}_{\left(S^{1} E\right)_{r e q}}$ in the formula for $\Lambda_{0}$.

In bases respecting the block decomposition induced from the direct sum, the linear part, other than the last term which lies in the upper right block, lies just below the diagonal blocks, and all blocks other than the upper right block and the diagonal and sub-diagonal blocks, are zero.

Moreover $N\left(T^{\mathrm{GL}(E)}\right) \subseteq \bar{\rho}_{A}\left(\mathbb{G}_{A}\right)$.

2.3. An equivariant representation of the permanent. We now give a minimal equivariant determinantal representation of perm $_{m}$. By Theorem 2.1, its size is $\left(\begin{array}{c}2 m \\ m\end{array}\right)-1$. For $e \otimes f \in E \otimes F^{*}$, let $S_{k}(e \otimes f):\left(S^{k} E\right)_{r e g} \otimes\left(S^{k} F^{*}\right)_{r e g} \rightarrow$ $\left(S^{k+1} E\right)_{r e g} \otimes\left(S^{k+1} F^{*}\right)_{\text {reg }}$ be multiplication by $e$ on the first factor and $f$ on the second followed by projection into $\left(S^{k+1} E\right)_{r e g} \otimes\left(S^{k+1} F^{*}\right)_{\text {reg. }}$. Equivalently,

$$
S_{k}:\left(E \otimes F^{*}\right) \rightarrow\left(\left(S^{k} E\right)_{r e g} \otimes\left(S^{k} F^{*}\right)_{r e g}\right)^{*} \otimes\left(S^{k+1} E\right)_{r e g} \otimes\left(S^{k+1} F^{*}\right)_{r e g}
$$

is the unique $N\left(T^{\mathrm{SL}(E)}\right) \times N\left(T^{\mathrm{SL}(F)}\right)$ equivariant inclusion.

Proposition 2.10. The following is an equivariant determinantal representation of perm ${ }_{m}$ : Let $\mathbb{C}^{n}=\oplus_{k=0}^{m-1}\left(S^{k} E\right)_{\text {reg }} \otimes\left(S^{k} F^{*}\right)_{\text {reg }}$, so $n=\left(\begin{array}{c}2 m \\ m\end{array}\right)-1 \sim 4^{m}$. Fix a linear isomorphism $S^{0} E \otimes S^{0} F^{*} \simeq\left(S^{m} E\right)_{\text {reg }} \otimes\left(S^{m} F^{*}\right)_{\text {reg. }}$. Set

$$
\Lambda_{0}=\sum_{k=1}^{m-1} \operatorname{Id}_{\left(S^{k} E\right)_{r e g} \otimes\left(S^{k} F^{*}\right)_{r e g}}
$$

and define

$$
\tilde{A}=(m !)^{\frac{-1}{n-m}} \Lambda_{0}+\sum_{k=0}^{m-1} S_{k}
$$

Then $(-1)^{m+1} \operatorname{perm}_{m}=\operatorname{det}_{n} \circ \tilde{A}$. In bases respecting the block structure induced by the direct sum, except for $S_{m-1}$, which lies in the upper right hand block, the linear part lies just below the diagonal block. 
2.4. Determinantal representations of quadrics. It will be instructive to examine other polynomials determined by their symmetry groups. Perhaps the simplest such is a nondegenerate quadratic form.

Let $Q=\sum_{j=1}^{s} x_{j} y_{j}$ be a non-degenerate quadratic form in $2 s$ variables. The polynomial $Q$ is characterized by its symmetries. By [MR04], if $s \geq 3$, the smallest determinantal representation of $Q$ is of size $s+1$ :

$$
\tilde{A}=\left(\begin{array}{cccc}
0 & -x_{1} & \cdots & -x_{s} \\
y^{1} & 1 & & \\
\vdots & & \ddots & \\
y^{s} & & & 1
\end{array}\right) .
$$

As described in $\S 4$, this representation respects about "half" the symmetry group $\mathbb{G}_{Q}$. We show in $\S 4$ that there is no size $s+1$ determinantal representation respecting $\mathbb{G}_{Q}$. However, Example 1.4 shows there is a size $2 s+1$ determinantal representation respecting $\mathbb{G}_{Q}$.

Proposition 2.11. Let $Q \in S^{2} \mathbb{C}^{M *}$ be a nondegenerate quadratic form, that is, a homogeneous polynomial of degree 2 . Then

$$
\operatorname{edc}(Q)=M+1 \text {. }
$$

2.5. Determinantal representations of the determinant. Although it may appear strange at first, one can ask for determinantal representations of $\operatorname{det}_{m}$. In this case, to get an interesting lower bound, we add a regularity condition motivated by Lemma 3.2:

Definition 2.12. Let $P \in S^{m} V^{*}$. A determinantal representation $\tilde{A}: V \longrightarrow$ $\mathcal{M}_{n}(\mathbb{C})$ is said to be regular if $\tilde{A}(0)$ has rank $n-1$.

Call the minimal size of a regular determinantal representation of $P$ the regular determinantal complexity of $P$ and denote it by $\operatorname{rdc}(P)$. Let $\operatorname{erdc}(P)$ denote the minimal size of a regular equivariant determinantal representation of $P$.

Any determinantal representation of perm $_{m}$ or a smooth quadric is regular, see $\S 3.3$. In contrast, the trivial determinantal representation of $\operatorname{det}_{m}$ is not regular; but this representation is equivariant so edc $\left(\operatorname{det}_{m}\right)=m$.

Theorem 2.13. $\operatorname{erdc}\left(\operatorname{det}_{m}\right)=\left(\begin{array}{c}2 m \\ m\end{array}\right)-1 \sim 4^{m}$.

As in the case of the permanent, we can get an exponential lower bound using only about half the symmetries of the determinant.

Theorem 2.14. Let $\tilde{A}_{m}: \mathcal{M}_{m}(\mathbb{C}) \longrightarrow \mathcal{M}_{n}(\mathbb{C})$ be a regular determinantal representation of $\operatorname{det}_{m}$ that respects $\mathrm{GL}(E)$. Then $n \geq 2^{m}-1$.

Moreover, there exists a regular determinantal representation of $\operatorname{det}_{m}$ that respects $\mathrm{GL}(E)$ of size $2^{m}-1$.

Remark 2.15. Normally when one obtains the same lower bound for the determinant as the permanent in some model it is discouraging for the model. However here there is an important difference due to the imposition of regularity for the determinant. We discuss this in further below Question 2.18. 
We now introduce notation to describe the regular determinantal representation of $\operatorname{det}_{m}$ that respects $\mathrm{GL}(E)$ of size $2^{m}-1$ mentioned in Theorem 2.14 .

Observe that $\left(S^{k} E\right)_{\text {reg }}$ is isomorphic to $\Lambda^{k} E$ as a $T^{\mathrm{GL}(E)}$-module but not as an $\mathfrak{S}_{m}$-module. The irreducible $\mathfrak{S}_{m}$-modules are indexed by partitions of $m$, write $[\pi]$ for the $\mathfrak{S}_{m}$-module associated to the partition $\pi$. Then as $\mathfrak{S}_{m}$-modules, $\left(S^{k} E\right)_{\text {reg }}=\oplus_{j=0}^{\min \{k, m-k\}}[m-j, j]$, while $\Lambda^{k} E=\left[m-k, 1^{k}\right] \oplus[m-$ $\left.k+1,1^{k-1}\right]$. In particular these spaces are not isomorphic as $N\left(T^{S L(E)}\right)$ modules.

Write $\mathcal{M}_{m}(\mathbb{C})=E \otimes F^{*}$. Let $f_{1}, \cdots, f_{m}$ be a basis of $F^{*}$. Let $e x_{k}$ denote exterior multiplication in $E$ :

$$
\begin{aligned}
e x_{k}: E & \longrightarrow\left(\Lambda^{k} E\right)^{*} \otimes\left(\Lambda^{k+1} E\right) \\
v & \mapsto\{\omega \mapsto v \wedge \omega\} .
\end{aligned}
$$

Proposition 2.16. The following is a regular determinantal representation of $\operatorname{det}_{m}$ that respects $\mathrm{GL}(E)$. Let $\mathbb{C}^{n}=\bigoplus_{j=0}^{m-1} \Lambda^{j} E$, so $n=2^{m}-1$ and $\operatorname{End}\left(\mathbb{C}^{n}\right)=\oplus_{0 \leq i, j \leq m-1} \operatorname{Hom}\left(\Lambda^{j} E, \Lambda^{i} E\right)$. Fix an identification $\Lambda^{m} E \simeq \Lambda^{0} E$. Set

$$
\Lambda_{0}=\sum_{k=1}^{m-1} \operatorname{Id}_{\Lambda^{k} E},
$$

and

$$
\tilde{A}=\Lambda_{0}+\sum_{k=0}^{m-1} e x_{k} \otimes f_{k+1} .
$$

Then $\operatorname{det}_{m}=\operatorname{det}_{n} \circ \tilde{A}$ if $m \equiv 1,2 \bmod 4$ and $\operatorname{det}_{m}=-\operatorname{det}_{n} \circ \tilde{A}$ if $m \equiv 0,3 \bmod 4$. In bases respecting the direct sum, the linear part, other than the last term which lies in the upper right block, lies just below the diagonal blocks, and all blocks other than the upper right, the diagonal and sub-diagonal are zero.

Note the similarity with the expression (11). This will be useful for proving the results about the determinantal representations of the permanent.

When $m=2$ this is

$$
\left(\begin{array}{ccc}
0 & -y_{2}^{2} & y_{1}^{2} \\
y_{1}^{1} & 1 & 0 \\
y_{2}^{1} & 0 & 1
\end{array}\right)
$$

agreeing with our earlier calculation of a rank four quadric. Note the minus sign in front of $y_{2}^{2}$ because $e x\left(e_{2}\right)\left(e_{1}\right)=-e_{1} \wedge e_{2}$.

For example, ordering the bases of $\Lambda^{2} \mathbb{C}^{3}$ by $e_{1} \wedge e_{2}, e_{1} \wedge e_{3}, e_{2} \wedge e_{3}$, the matrix for $\operatorname{det}_{3}$ is

$$
\left(\begin{array}{ccccccc}
0 & 0 & 0 & 0 & y_{3}^{3} & -y_{2}^{3} & y_{1}^{3} \\
y_{1}^{1} & 1 & & & & & \\
y_{2}^{1} & & 1 & & & & \\
y_{3}^{1} & & & 1 & & & \\
& -y_{2}^{2} & y_{1}^{2} & 0 & 1 & & \\
& -y_{3}^{2} & 0 & y_{1}^{2} & & 1 & \\
& 0 & -y_{3}^{2} & y_{2}^{2} & & & 1
\end{array}\right)
$$


We now give a regular equivariant determinantal representation of $\operatorname{det}_{m}$. Let $E X_{k}$ denote the exterior multiplication

$$
\begin{aligned}
E X_{k}: E \otimes F^{*} & \longrightarrow\left(\Lambda^{k} E \otimes \Lambda^{k} F^{*}\right)^{*} \otimes\left(\Lambda^{k+1} E \otimes \Lambda^{k+1} F^{*}\right) \\
e \otimes f & \mapsto\{\omega \otimes \eta \mapsto e \wedge \omega \otimes f \wedge \eta\},
\end{aligned}
$$

Proposition 2.17. The following is an equivariant regular determinantal representation of $\operatorname{det}_{m}$. Let $\mathbb{C}^{n}=\bigoplus_{j=0}^{m-1} \Lambda^{j} E \otimes \Lambda^{j} F^{*}$, so $n=\left(\begin{array}{c}2 m \\ m\end{array}\right)-1 \sim 4^{m}$ and $\operatorname{End}\left(\mathbb{C}^{n}\right)=\oplus_{0 \leq i, j \leq m} \operatorname{Hom}\left(\Lambda^{j} E \otimes \Lambda^{j} F^{*}, \Lambda^{i} E \otimes \Lambda^{i} F^{*}\right)$. Fix an identification $\Lambda^{m} E \otimes \Lambda^{m} F^{*} \simeq \Lambda^{0} E \otimes \Lambda^{0} F^{*}$. Set

$$
\Lambda_{0}=\sum_{k=1}^{m-1} \operatorname{Id}_{\Lambda^{k} E \otimes \Lambda^{k} F^{*}}
$$

and define

$$
\tilde{A}=(m !)^{\frac{-1}{n-m}} \Lambda_{0}+\sum_{k=0}^{m-1} E X_{k}
$$

Then $(-1)^{m+1} \operatorname{det}_{m}=\operatorname{det}_{n} \circ \tilde{A}$.

Comparing Theorems 2.1 and 2.13, Theorems 2.8 and 2.14, Propositions 2.9 and 2.16 and Propositions 2.10 and 2.17, one can see that $\operatorname{det}_{m}$ and $\operatorname{perm}_{m}$ have the same behavior relatively to equivariant regular determinantal representations. This prompts the question: What is the regular determinantal complexity of the determinant? In particular:

Question 2.18. Let $\operatorname{rdc}\left(\operatorname{det}_{m}\right)$ be the smallest value of $n$ such that there exist affine linear maps $\tilde{A}_{m}: \mathbb{C}^{m^{2}} \rightarrow \mathbb{C}^{n^{2}}$ such that

$$
\operatorname{det}_{m}=\operatorname{det}_{n} \circ \tilde{A}_{m} \text { and } \operatorname{rank} \tilde{A}(0)=n-1 .
$$

What is the growth of $\operatorname{rdc}\left(\operatorname{det}_{m}\right)$ ?

Because of the symmetries of $\operatorname{det}_{n}$, this question might be easier than determining the growth of $\operatorname{dc}\left(\operatorname{perm}_{m}\right)$. If $\operatorname{rdc}\left(\operatorname{det}_{m}\right)$ grows faster than a polynomial, this would provide a path to proving Valiant's conjecture by trying to transport the bound to the permanent via the Howe-Young duality functor [AW07], which guided the proofs of the permanental cases in this article and enabled the computation of the linear strand of the minimal free resolution of the ideal generated by subpermanents in [ELSW15]. Even if this fails, it would still refocus research towards the large singular locus of $\left\{\operatorname{det}_{n}=0\right\}$. If $\operatorname{rdc}\left(\operatorname{det}_{m}\right)$ grows polynomially, then one could still try to transport the bound to the permanent. If one is unable to do so, the breakdown of the method could give better insight to the difference between $\operatorname{det}_{m}$ and perm $_{m}$. Polynomial growth would also give a negative answer to the analog of Question 2.2 for the determinant.

2.6. Overview. In $\S 3$ we establish basic facts about determinantal representations and review results about algebraic groups. The proofs of the results are then presented in increasing order of difficulty, beginning with the easy case of quadrics in $\S 4$, then the case of the determinant in $\S 5$, and concluding with the permanent in $\S 6$. 
2.7. Acknowledgments. The seed for this article was planted during the Fall 2014 semester program Algorithms and Complexity in Algebraic Geometry at the Simons Institute for the Theory of Computing, UC Berkeley. Most of the work was done when the Landsberg was a guest of Ressayre and Pascal Koiran. Landsberg thanks his hosts as well as U. Lyon and ENS Lyon for their hospitality and support. We thank: Christian Ikenmeyer for useful discussions about equivariant determinantal presentations of the permanent and Grenet's algorithm, Josh Grochow, Christian Ikenmeyer and Shrawan Kumar for useful suggestions for improving the exposition, Jérôme Germoni for mentioning the existence of the spin symmetric group, Michael Forbes for suggesting Example 2.5, and Avi Wigderson for suggesting Example 2.6.

\section{Preliminaries on Symmetries}

Throughout this section $P \in S^{m} V^{*}$ is a polynomial and

$$
\tilde{A}=\Lambda+A: V \longrightarrow \mathcal{M}_{n}(\mathbb{C})
$$

is a determinantal representation of $P$.

3.1. Notation. For an affine algebraic group $G, G^{\circ}$ denotes the connected component of the identity. The homomorphisms from $G$ to $\mathbb{C}^{*}$ are called characters of $G$. They form an abelian group denoted by $X(G)$. The law in $X(G)$ is denoted additively (even if it comes from multiplication in $\mathbb{C}^{*}$ ). If $G \simeq\left(\mathbb{C}^{*}\right)^{\times r}$ is a torus then $X(G) \simeq \mathbb{Z}^{r}$.

For a vector space $V, \mathbb{P} V$ is the associated projective space. For a polynomial $P$ on $V,\{P=0\}=\{x \in V: P(x)=0\}$ denotes its zero set, which is an affine algebraic variety. For $v \in V$, the differential of $P$ at $v$ is denoted $d_{v} P \in V^{*}$ and $\{P=0\}_{\text {sing }}=\left\{x \in V: P(x)=0\right.$ and $\left.d_{x} P=0\right\}$ denotes the singular locus of $\{P=0\}$. Note that we do not consider the reduced algebraic variety, in particular if $P$ is a square $\{P=0\}_{\text {sing }}=\{P=0\}$.

3.2. Representations of $\mathbb{G}_{A}$. The following observation plays a key role:

Lemma 3.1. $\bar{\rho}_{A}\left(\mathbb{G}_{A}\right) \subset \mathbb{G}_{P}$. Moreover, for any $g \in \mathbb{G}_{A}, \chi_{\operatorname{det}_{n}}(g)=\chi_{P}\left(\bar{\rho}_{A}(g)\right)$.

Proof. Let $g \in \mathbb{G}_{A}$ and $v \in V$. Then

$$
\begin{aligned}
\left(\bar{\rho}_{A}(g) P\right)(v) & =P\left(\bar{\rho}_{A}(g)^{-1} v\right) \\
& =\operatorname{det}_{n}\left(\Lambda+A\left(A^{-1} g^{-1} A(v)\right)\right) \\
& =\operatorname{det}_{n}\left(g^{-1}(\Lambda+A(v))\right. \\
& =\left(g \operatorname{det}_{n}\right)(\tilde{A}(v)) \\
& =\chi_{\operatorname{det}_{n}}(g) \operatorname{det}_{n}(\tilde{A}(v)) \\
& =\chi_{\operatorname{det}_{n}}(g) P(v) .
\end{aligned}
$$

The lemma follows.

\subsection{Normal form for $\Lambda$.}

Lemma 3.2. [vzG87] Let $P \in S^{m} V^{*}$ be a polynomial. If $\operatorname{codim}(\{P=$ $\left.0\}_{\text {sing }}, V\right) \geq 5$, then any determinantal representation $\tilde{A}$ of $P$ is regular. 
Proof. Consider the affine variety $\mathcal{D}_{n}=\left\{\operatorname{det}_{n}=0\right\}$. The singular locus of $\mathcal{D}_{n}$ is the set of matrices of rank at most $n-2$, and hence has codimension 4 in $\mathcal{M}_{n}(\mathbb{C})$.

For any $v \in V$, we have $d_{v} P=d_{v}\left(\operatorname{det}_{n} \circ \tilde{A}\right)=d_{\tilde{A}(v)}\left(\operatorname{det}_{n}\right) \circ A$. In particular, if $\tilde{A}(v) \in\left(\mathcal{D}_{n}\right)_{\text {sing }}$ then $v \in\{P=0\}_{\text {sing }}$.

But the set of $v$ such that $\operatorname{rk}(\tilde{A}(v)) \leq n-2$ is either empty, or its codimension is at most 4 . The assumption of the lemma implies that $\operatorname{rk}(\tilde{A}(v)) \geq n-1$ for any $v \in V$. In particular $\operatorname{rk}(\tilde{A}(0))=n-1$.

In [vzG87], von zur Gathen showed that $\operatorname{codim}\left(\left\{\text { perm }_{m}=0\right\}_{\text {sing }}, \mathbb{C}^{m^{2}}\right) \geq 5$.

Let $\Lambda_{n-1} \in \mathcal{M}_{n}(\mathbb{C})$ be the matrix with 1 in the $n-1$ last diagonal entries and 0 elsewhere. Any determinantal representation $\tilde{A}$ of $P$ of size $n$ with $\operatorname{rank}(\tilde{A}(0))=n-1$ can be transformed (by multiplying on the left and right by constant invertible matrices) to a determinantal representation of $P$ satisfying $\tilde{A}(0)=\Lambda_{n-1}$.

3.4. An auxiliary symmetry group. Recall the group $\mathbb{G}_{\operatorname{det}_{n}} \subset \mathrm{GL}_{n^{2}}$ from Equation (4). The following group plays a central role in the study of regular equivariant determinantal representations:

$$
\mathbb{G}_{\operatorname{det}_{n}, \Lambda_{n-1}}=\left\{g \in \mathbb{G}_{\operatorname{det}_{n}} \mid g \cdot \Lambda_{n-1}=\Lambda_{n-1}\right\} .
$$

Let $\mathbb{H} \subset \mathbb{C}^{n}$ denote the image of $\Lambda_{n-1}$ and $\ell_{1} \in \mathbb{C}^{n}$ its kernel. Write $\ell_{2}$ for $\ell_{1}$ in the target $\mathbb{C}^{n}$. Then $\mathcal{M}_{n}(\mathbb{C})=\left(\ell_{1} \oplus \mathbb{H}\right)^{*} \otimes\left(\ell_{2} \oplus \mathbb{H}\right)$. Let transp $\in \operatorname{GL}\left(\mathcal{M}_{n}(\mathbb{C})\right)$ denote the transpose.

Lemma 3.3. The group $\mathbb{G}_{\operatorname{det}_{n}, \Lambda_{n-1}}$ is

$$
\left\{M \mapsto\left(\begin{array}{cc}
\lambda_{2} & 0 \\
v_{2} & g
\end{array}\right) M\left(\begin{array}{cc}
1 & \phi_{1} \\
0 & g
\end{array}\right)^{-1} \mid g \in \mathrm{GL}(\mathbb{H}), v_{2} \in \mathbb{H}, \phi_{1} \in \mathbb{H}^{*}, \lambda_{2} \in \mathbb{C}^{*}\right\} \cdot\langle\text { transp }\rangle .
$$

In particular, it is isomorphic to

$$
\left[\mathrm{GL}\left(\ell_{2}\right) \times \mathrm{GL}(\mathbb{H}) \ltimes\left(\mathbb{H} \oplus \mathbb{H}^{*} \otimes \ell_{2}\right)\right] \rtimes \mathbb{Z}_{2}
$$

Proof. First note that $\operatorname{transp}\left(\Lambda_{n-1}\right)=\Lambda_{n-1}$, so it is sufficient to determine the stabilizer of $\Lambda_{n-1}$ in $\mathbb{G}_{\operatorname{det}_{n}}^{\circ}$. Let $A, B \in \mathrm{GL}_{n}(\mathbb{C})$ such that $A \Lambda_{n-1} B^{-1}=$ $\Lambda_{n-1}$. Since $A$ stabilizes the image of $\Lambda_{n-1}$ and $B$ stabilizes the Kernel of $\Lambda_{n-1}$ we have

$$
A=\left(\begin{array}{cc}
\lambda_{2} & 0 \\
v_{2} & g_{2}
\end{array}\right) \quad \text { and } \quad B=\left(\begin{array}{cc}
\lambda_{1} & \phi_{1} \\
0 & g_{1}
\end{array}\right)
$$

for some $\lambda_{1} \in \mathrm{GL}\left(\ell_{1}\right), \lambda_{2} \in \mathrm{GL}\left(\ell_{2}\right), g_{1}, g_{2} \in \mathrm{GL}(\mathbb{H}), v_{2} \in \mathbb{H}$ and $\phi_{1} \in \mathbb{H}^{*}$. The identity $A \Lambda_{n-1} B^{-1}=\Lambda_{n-1}$ is now equivalent to $g_{1}=g_{2}$. Multiplying $A$ and $B$ by $\lambda_{1}^{-1} \operatorname{Id}_{n}$ gives the result.

3.5. Facts about complex algebraic groups. Let $G$ be an affine complex algebraic group. The group $G$ is

- reductive if every $G$-module may be decomposed into a direct sum of irreducible $G$-modules.

- unipotent if it is isomorphic to a subgroup of the group $U_{n}$ of upper triangular matrices with 1's on the diagonal. 
Given a complex algebraic group $G$, there exists a maximal normal unipotent subgroup $R^{u}(G)$, called the unipotent radical. The quotient $G / R^{u}(G)$ is reductive. Moreover there exists subgroups $L$ in $G$ such that $G=R^{u}(G) L$. In particular such $L$ are reductive. Such a subgroup $L$ is not unique, but any two such are conjugate in $G$ (in fact by an element of $R^{u}(G)$ ). Such a subgroup $L$ is called a Levi factor of $G$. A good reference is [OV90, Thm. 4. Chap. 6].

Malcev's theorem (see, e.g., [OV90, Thm. 5. Chap. 6]) states that fixing a Levi subgroup $L \subset G$ and given any reductive subgroup $H$ of $G$, there exists $g \in R^{u}(G)$ such that $g H g^{-1} \subseteq L$.

For example, when $G$ is a parabolic subgroup, e.g. $G=\left(\begin{array}{cc}* & * \\ 0 & *\end{array}\right)$, we have $L=\left(\begin{array}{cc}* & 0 \\ 0 & *\end{array}\right)$ and $R^{u}(G)=\left(\begin{array}{cc}\operatorname{Id}_{a} & * \\ 0 & \operatorname{Id}_{b}\end{array}\right)$.

A more important example for us is $R^{u}\left(\mathbb{G}_{\operatorname{det}_{n}, \Lambda_{n-1}}\right)=\left(\mathbb{H} \oplus \mathbb{H}^{*} \otimes \ell_{2}\right)$ and a Levi subgroup is $L=\left(\mathrm{GL}\left(\ell_{2}\right) \times \mathrm{GL}(\mathbb{H})\right) \rtimes \mathbb{Z}_{2}$.

3.6. Outline of the proofs of lower bounds. Let $P \in S^{m} V^{*}$ be either a quadric, a permanent or a determinant. Say a regular representation $\tilde{A}$ respects some $G \subseteq \mathbb{G}_{P}$. We may assume that $\tilde{A}(0)=\Lambda_{n-1}$.

The first step consists in lifting $G$ to $\mathbb{G}_{A}$. More precisely, in each case we construct a reductive subgroup $\tilde{G}$ of $\mathbb{G}_{A}$ such that $\bar{\rho}_{A}: \tilde{G} \longrightarrow G$ is finite and surjective. In a first reading, it is relatively harmless to assume that $\tilde{G} \simeq G$. Then, using Malcev's theorem, after possibly conjugating $\tilde{A}$, we may assume that $\tilde{G}$ is contained in $\left(\mathrm{GL}\left(\ell_{2}\right) \times \mathrm{GL}(\mathbb{H})\right) \rtimes \mathbb{Z}_{2}$. Up to considering an index two subgroup of $\tilde{G}$ if necessary, we assume that $\tilde{G}$ is contained in $\operatorname{GL}\left(\ell_{2}\right) \times \mathrm{GL}(\mathbb{H})$ (with the notation of Lemma 3.3).

Now, both $\mathcal{M}_{n}(\mathbb{C})=\left(\ell_{1} \oplus \mathbb{H}\right)^{*} \otimes\left(\ell_{2} \oplus \mathbb{H}\right)$ and $V\left(\right.$ via $\left.\bar{\rho}_{A}\right)$ are $\tilde{G}$-modules. Moreover, $A$ is an equivariant embedding of $V$ in $\mathcal{M}_{n}(\mathbb{C})$. This turns out to be a very restrictive condition.

Write

$$
\mathcal{M}_{n}(\mathbb{C})=\left(\begin{array}{cc}
\ell_{1}^{*} \otimes \ell_{2} & \mathbb{H}^{*} \otimes \ell_{2} \\
\ell_{1}^{*} \otimes \mathbb{H} & \mathbb{H}^{*} \otimes \mathbb{H}
\end{array}\right), \quad \Lambda_{n-1}=\left(\begin{array}{cc}
0 & 0 \\
0 & \operatorname{Id}_{\mathbb{H}}
\end{array}\right)
$$

If $m \geq 2$ the $\ell_{1}^{*} \otimes \ell_{2}$ coefficient of $\tilde{A}$ has to be zero. Then, since $P \neq 0$, the projection of $A(V)$ on $\ell_{1}^{*} \otimes \mathbb{H} \simeq \mathbb{H}$ has to be non-zero. We thus have a $G$ submodule $\mathbb{H}_{1} \subset \mathbb{H}$ isomorphic to an irreducible submodule of $V$. A similar argument shows that there must be another irreducible $G$-submodule $\mathbb{H}_{2} \subset \mathbb{H}$ such that an irreducible submodule of $V$ appears in $\mathbb{H}_{1}^{*} \otimes \mathbb{H}_{2}$.

In each case, we can construct a sequence of irreducible sub- $\tilde{G}$-modules $\mathbb{H}_{k}$ of $\mathbb{H}$ satisfying very restrictive conditions. This allows us to get our lower bounds.

To prove the representations $\tilde{A}$ actually compute the polynomials we want, in the case $G=\mathbb{G}_{P}$, we first check that $\mathbb{G}_{P}$ is contained in the image of $\bar{\rho}_{A}$. Since $P$ is characterized by its symmetries, we $\operatorname{deduce}$ that $\operatorname{det}_{n} \circ \tilde{A}$ is a scalar multiple of $P$. We then specialize to evaluating on the diagonal 
matrices in $\mathcal{M}_{m}(\mathbb{C})$ to determine this constant, proving in particular that it is non-zero.

\section{Symmetric Determinantal RePRESEntations of QUADRICS}

We continue the notation of $\S 2.4$, in particular $Q \in S^{2} \mathbb{C}^{2 s}$ is a nondegnerate quadric.

Let $\tau: \mathbb{C}^{*} \times O(2 s) \longrightarrow G L(2 s)$ be given by $(\lambda, M) \mapsto \lambda M$. The image of $\tau$ is the group $\mathbb{G}_{Q}$ and $\chi_{Q} \circ \tau(\lambda, M)=\lambda^{-2}$.

In the expression $(13)$ we have $\mathbb{G}_{A}=\left(\mathrm{GL}\left(\ell_{2}\right) \times \mathrm{GL}(\mathbb{H})\right) \rtimes \mathbb{Z} / 2 \mathbb{Z}$. Since $\rho_{A}\left(\mathbb{G}_{A}^{\circ}\right)$ is a proper subgroup of $\mathbb{G}_{Q}$, this determinantal representation is not equivariant.

More generally, let $\tilde{B}$ be any determinantal representation of $Q$ of size $s+1$. By Lemma $3.2 \operatorname{rank}(\Lambda)=s$. Then the dimension of $\mathbb{G}_{B}$ cannot exceed that of $\mathbb{G}_{\operatorname{det}_{s+1}, \Lambda_{s}}$, which is $(s+1)^{2}$ by Lemma 3.3. But the dimension of $\mathbb{G}_{Q}$ is $2 s^{2}-s+1$. Hence the representation cannot respect the symmetries. (This argument has to be refined when $s=3$, observing that the unipotent radical of $\mathbb{G}_{\operatorname{det}_{s+1}, \Lambda_{s}}$ is contained in the kernel of $\rho_{A}$.)

Nonetheless, in the case of quadrics, the smallest presentation $A$ respects about "half" the symmetry, as was the case in Example 2.5. We will see this again with perm $_{3}$ but so far have no explanation.

Proof of Proposition 2.11. Let $\tilde{A}=\Lambda+A$ be a equivariant determinantal representation of $Q$. By Lemma 3.2, one may assume that $\tilde{A}(0)=\Lambda_{n-1}$.

We now construct an analog $L$ of the group $\tilde{G}$ mentioned in $\S 3.6$. Start with $H=\bar{\rho}_{A}{ }^{-1}\left(\mathbb{G}_{Q}\right)$. Consider a Levi decomposition $H=R^{u}(H) L$. Then $\bar{\rho}_{A}\left(R^{u}(H)\right)$ is a normal unipotent subgroup of $\mathbb{G}_{Q}$. Since $\mathbb{G}_{Q}$ is reductive this implies that $R^{u}(H)$ is contained in the kernel of $\bar{\rho}_{A}$. In particular, $\bar{\rho}_{A}(L)=\mathbb{G}_{Q}$. Since $\mathbb{G}_{Q}$ is connected, $\bar{\rho}_{A}\left(L^{\circ}\right)=\mathbb{G}_{Q}$.

By construction $L$ is a reductive subgroup of $\mathbb{G}_{\operatorname{det}_{n}, \Lambda_{n-1}}$. By Malcev's theorem, possibly after conjugating $\tilde{A}$, we may and will assume that $L$ is contained in $\left(\mathrm{GL}\left(\ell_{2}\right) \times \mathrm{GL}(\mathbb{H})\right) \rtimes \mathbb{Z}_{2}$. In particular $L^{\circ}$ is contained in $\operatorname{GL}\left(\ell_{2}\right) \times$ $\mathrm{GL}(\mathbb{H})$ and $\bar{\rho}_{A}\left(L^{\circ}\right)=\mathbb{G}_{Q}$.

Observe that $A(V)$ is an irreducible $L^{\circ}$-submodule of $\mathcal{M}_{n}(\mathbb{C})$ isomorphic to $V$. Moreover, the action of $L^{\circ}$ respects the decomposition

$$
\mathcal{M}_{n}(\mathbb{C})=\ell_{1}^{*} \otimes \ell_{2} \oplus \ell_{1}^{*} \otimes \mathbb{H} \oplus \mathbb{H}^{*} \otimes \ell_{2} \oplus \mathbb{H}^{*} \otimes \mathbb{H} .
$$

The projection of $A(V)$ on $\ell_{1}^{*} \otimes \ell_{2}$ has to be zero, since it is $L^{\circ}$-equivariant. Hence

$$
A(V) \subset \ell_{1}^{*} \otimes \mathbb{H} \oplus \mathbb{H}^{*} \otimes \ell_{2} \oplus \mathbb{H}^{*} \otimes \mathbb{H} .
$$

In matrices:

$$
A(V) \subset\left(\begin{array}{cc}
0 & \mathbb{H}^{*} \otimes \ell_{2} \\
\ell_{1}^{*} \otimes \mathbb{H} & \mathbb{H}^{*} \otimes \mathbb{H}
\end{array}\right)
$$

Thus for the determinant to be non-zero, we need the projection to $\ell_{1}^{*} \otimes \mathbb{H}$ to be non-zero. Thus it must contain at least one copy of $V$. In particular $\operatorname{dim}(\mathbb{H}) \geq \operatorname{dim}(V)$; the desired inequality. 


\section{Proofs of the determinantal Representations of the DETERMINANT}

Recall our notations that $E, F$ are complex vector spaces of dimension $m$ and we have an identification $E \otimes F^{*} \simeq \operatorname{Hom}(F, E)$. A linear map $u: F \rightarrow E$ induces linear maps

$$
\begin{gathered}
u^{\wedge k}: \Lambda^{k} F \rightarrow \Lambda^{k} E \\
v_{1} \wedge \cdots \wedge v_{k} \mapsto u\left(v_{1}\right) \wedge \cdots \wedge u\left(v_{k}\right) .
\end{gathered}
$$

In the case $k=m, u^{\wedge m}$ is called the determinant of $u$ and we denote it $\operatorname{Det}(u) \in \Lambda^{m} F^{*} \otimes \Lambda^{m} E$. The map

$$
\begin{aligned}
E \otimes F^{*}=\operatorname{Hom}(F, E) & \longrightarrow \Lambda^{m} F^{*} \otimes \Lambda^{m} E \\
u & \longmapsto \operatorname{Det}(u)
\end{aligned}
$$

is polynomial, homogeneous of degree $m$, and equivariant for the natural action of $\mathrm{GL}(E) \times \mathrm{GL}(F)$.

The transpose of $u$ is

$$
\begin{aligned}
u^{T}: E^{*} & \longrightarrow F^{*}, \\
\varphi & \longmapsto \varphi \circ u .
\end{aligned}
$$

Hence $u^{T} \in F^{*} \otimes E$ is obtained from $u$ by switching $E$ and $F^{*}$, and $\operatorname{Det}\left(u^{T}\right) \epsilon$ $\Lambda^{m} E \otimes \Lambda^{m} F^{*}$. Moreover, $\operatorname{Det}\left(u^{T}\right)=\operatorname{Det}(u)^{T}$.

Proof of Proposition 2.16. Set $P=\operatorname{det}_{n} \circ \tilde{A}$. To analyze the action of GL $(E)$ on $\tilde{A}$, reinterpret $\mathbb{C}^{n *} \otimes \mathbb{C}^{n}$ without the identification $\Lambda^{0} E \simeq \Lambda^{m} E$ as $\left(\oplus_{j=0}^{m-1} \Lambda^{j} E\right)^{*} \otimes\left(\oplus_{i=1}^{m} \Lambda^{i} E\right)$.

For each $u \in E \otimes F^{*}$, associate to $\tilde{A}(u)$ a linear map $\tilde{a}(u): \oplus_{j=0}^{m-1} \Lambda^{j} E \rightarrow$ $\oplus_{i=1}^{m} \Lambda^{i} E$. Then $\operatorname{Det}(\tilde{a}(u)) \in \Lambda^{n}\left(\oplus_{j=0}^{m-1} \Lambda^{j} E^{*}\right) \otimes \Lambda^{n}\left(\oplus_{i=1}^{m} \Lambda^{i} E\right)$. This space may be canonically identified as a $G L(E)$-module with $\Lambda^{0} E^{*} \otimes \Lambda^{m} E \simeq \Lambda^{m} E$. (The identification $\Lambda^{0} E \simeq \Lambda^{m} E$ allows one to identify this space with $\mathbb{C}$.) Using the maps (17), we get $\mathrm{GL}(E)$-equivariant maps

$$
E \otimes F^{*} \stackrel{\tilde{a}}{\longrightarrow} \operatorname{Hom}\left(\oplus_{j=0}^{m-1} \Lambda^{j} E, \oplus_{i=1}^{m} \Lambda^{i} E\right) \stackrel{\mathcal{D} e t}{\longrightarrow} \Lambda^{m} E .
$$

Hence for all $u \in E \otimes F^{*}$ and all $g \in \operatorname{GL}(E)$,

$$
\begin{aligned}
\operatorname{Det}\left(\tilde{a}\left(g^{-1} u\right)\right) & =(g \cdot \operatorname{Det})(\tilde{a}(u)) \\
& =\operatorname{det}(g)^{-1} \operatorname{Det}(\tilde{a}(u)) .
\end{aligned}
$$

Equation (18) shows that GL(E) is contained in the image of $\bar{\rho}_{A}$.

Equation (18) also proves that $P$ is a scalar (possibly zero) multiple of the determinant. Consider $P\left(\operatorname{Id}_{m}\right)=\operatorname{det}_{n}\left(\tilde{A}\left(\operatorname{Id}_{m}\right)\right)$. Perform a Laplace expansion of this large determinant: there is only one non-zero expansion term, so $P$ is the determinant up to a sign.

To see the sign is as asserted in Proposition 2.16, specialize to the diagonal matrices, then there is just one term. Note that $y_{i}^{i}$ appears in the large matrix with the sign $(-1)^{i+1}$. Thus the total contribution of these signs is $(-1)$ if $m \equiv 2,3 \bmod 4$ and $(+1)$ if $m \equiv 0,1 \bmod 4$. 
The $k$-th block of $\Lambda$ contributes a sign of $(-1)\left(\begin{array}{c}m \\ k\end{array}\right)-1$ if we perform a left to right Laplace expansion, except for the last which contributes $(1)^{m-1}$. (The terms are negative because the $I d_{\left(\begin{array}{c}m \\ k\end{array}\right)-1}$ will begin in the left-most column each time, but, except for the last block, it begins in the second row.) Thus the total contribution of $\Lambda$ to the sign is $(-1)^{\sum_{k=1}^{m-2}\left[\left(\begin{array}{c}m \\ k\end{array}\right)-1\right]}=1$.

The slot of each $\pm y_{i}^{i}$ except $y_{m}^{m}$ (whose slot always contributes positively in the Laplace expansion) contributes a $(-1)^{\left(\begin{array}{c}m \\ i-1\end{array}\right)}$ (it is always left-most, but appears $\left(\begin{array}{c}m \\ i-1\end{array}\right)+1$ slots below the top in the remaining matrix). Thus the total contribution from these slots is $(-1)^{\sum_{i=0}^{m-2}\left(\begin{array}{c}m \\ i\end{array}\right)}=(-1)^{2^{m}-m-1}=(-1)^{m+1}$.

Thus the total sign is -1 if $m \equiv 0,3 \bmod 4$ and +1 if $m \equiv 1,2 \bmod 4$.

Proof of Theorem 2.13. Let $\tilde{A}=\Lambda+A: \mathcal{M}_{m}(\mathbb{C}) \rightarrow \mathcal{M}_{n}(\mathbb{C})$ be a regular determinantal representation of $\operatorname{det}_{m}$ that respects $\mathrm{GL}(E)$. It remains to prove that $n \geq 2^{m}-1$.

We may assume $\Lambda=\Lambda_{n-1}$. As in the proof of Proposition 2.11, after possibly conjugating $\tilde{A}$, we construct a connected reductive subgroup $L$ of $\operatorname{GL}\left(\ell_{2}\right) \times \mathrm{GL}(\mathbb{H})$ mapping onto $\mathrm{GL}(E)$ by $\bar{\rho}_{A}$.

We have an action of $L$ on $\mathcal{M}_{n}(\mathbb{C})$, but we would like to work with $\mathrm{GL}(E)$. Towards this end, there exists a finite cover $\tau: \tilde{L} \longrightarrow L$ that is isomorphic to the product of a torus and a product of simple simply connected groups. In particular there exists a subgroup of $\tilde{L}$ isomorphic to $\mathbb{C}^{*} \times \mathrm{SL}(E)$ such that $\bar{\rho}_{A} \circ \tau\left(\mathbb{C}^{*} \times \mathrm{SL}(E)\right)=\mathrm{GL}(E)$. The group $\mathbb{C}^{*} \times \mathrm{SL}(E)$ acts trivially on $\ell_{1}$, on $\ell_{2}$ (by some character) and on $\mathbb{H}$. It acts on $\mathcal{M}_{n}(\mathbb{C})=\left(\ell_{1}^{*} \oplus \mathbb{H}\right) \otimes\left(\ell_{2} \oplus \mathbb{H}\right)$ accordingly.

The $\mathbb{C}^{*} \times \mathrm{SL}(E)$-module $A(V)$ is isomorphic to the sum of $m$ copies of $E$, and $E$ is an irreducible $\mathbb{C}^{*} \times \operatorname{SL}(E)$-module. In particular its equivariant projection on $\ell_{1}^{*} \otimes \ell_{2}$ is zero, which implies that the $(1,1)$ entry of the matrix of $\tilde{A}$ (in adapted bases) is zero. Consider the equivariant projection of $A(V)$ on $\ell_{1}^{*} \otimes \mathbb{H}$. This projection in bases goes to the remainder of the first column. It must be non-zero or $\operatorname{det}_{n} \circ \tilde{A}$ will be identically zero. Since it is equivariant, $\ell_{1}^{*} \otimes \mathbb{H} \simeq \mathbb{H}$ must contain $E$ as a $\mathbb{C}^{*} \times \mathrm{SL}(E)$-module. Similarly, examining the first row, $\mathbb{H}^{*} \otimes \ell_{2}$ has to contain $E$ as a $\mathbb{C}^{*} \times \mathrm{SL}(E)$-module.

If $m=2$, it is possible that $\mathbb{H} \simeq E$ and $\mathbb{H} \otimes \ell_{2} \simeq E^{*}$. In this case, $\operatorname{det}_{2}$ is a quadratic form, and we recover its determinantal representation of size 3 .

Assume now that $m \geq 3$, in particular that $E$ and $E^{*}$ are not isomorphic as $\mathrm{SL}(E)$-modules. We just proved that $\mathbb{H}$ must contain a subspace isomorphic to $E$, say $\mathbb{H}_{1}$. Since $\mathbb{H}_{1}^{*} \otimes \ell_{2}$ is an irreducible $\operatorname{SL}(E)$-module and not isomorphic to $E$, the projection of $A(V)$ on this factor is zero.

Choose a $\mathbb{C}^{*} \times \mathrm{SL}(E)$-stable complement $S_{1}$ to $\mathbb{H}_{1}$ in $\mathbb{H}$. If the projection of $A(V)$ to the block $\mathbb{H}_{1}^{*} \otimes S_{1}$ is zero, by expanding the columns corresponding to $\mathbb{H}_{1}^{*}$, one sees that $\operatorname{det}_{m}$ is equal to the determinant in $\left(\ell_{1} \oplus S_{1}\right)^{*} \otimes\left(\ell_{2} \otimes S_{1}\right)$, and we can restart the proof with $S_{1}$ in place of $\mathbb{H}$.

So assume that the projection of $A(V)$ onto the block $\mathbb{H}_{1}^{*} \otimes S_{1}$ is non-zero. Then there must be some irreducible $\left(\mathbb{C}^{*} \times \mathrm{SL}(E)\right)$-submodule $\mathbb{H}_{2}$ such that $\mathbb{H}_{1}^{*} \otimes \mathbb{H}_{2}$ contains $E$ as a submodule. Continuing, we get a sequence of simple $\left(\mathbb{C}^{*} \times \mathrm{SL}(E)\right)$-submodules $\mathbb{H}_{1}, \ldots, \mathbb{H}_{k}$ of $\mathbb{H}$ such that $E$ is a submodule of $\mathbb{H}_{i}^{*} \otimes \mathbb{H}_{i+1}$ and of $\ell_{2} \otimes \mathbb{H}_{k}^{*}$. 
The situation is easy to visualize with Young diagrams. The irreducible polynomial representations of $\mathrm{GL}(E)$ are indexed by partitions, and irreducible representations of $\mathrm{SL}(E)$ by equivalence classes of partitions where $\pi \pm\left(c^{m}\right) \sim \pi$, and to a partition $\pi=\left(p_{1}, \cdots, p_{m-1}\right)$ we associate a Young diagram, a collection of left-aligned boxes with $p_{j}$ boxes in the $j$-th row. For example, the Young diagram for $\pi=(2,1,1)=:\left(2,1^{2}\right)$ is

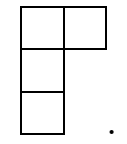

For example, as an $S L(E)$-module, $E$ (resp. $E^{*}$ ) corresponds the class of to a single box (resp. a column of $m-1$ boxes). (As a $G L(E)$-module, $E^{*}$ corresponds to a diagram with -1 boxes.) The Pieri formula implies that $E \subset S_{\pi} E^{*} \otimes S_{\mu} E$ if and only if the diagram of $\mu$ is obtained from the diagram of $\pi$ by adding a box. Then the sequence of Young diagrams associated to the irreducible $\mathrm{SL}(E)$ modules $\mathbb{H}_{i}$ start with one box, and increases by one box at each step. Thus we must have $\mathbb{H}_{k}$ associated to $\pi=\left(c^{m-1}, c-1\right)$ for some $c$. To have the proper $\mathbb{C}^{*}$-action, we choose the action on $\ell_{2}$ to cancel the $(c-1) \times m$ box. We deduce that $\left(\mathbb{H}_{1}, \cdots, \mathbb{H}_{k}\right)=\left(\Lambda^{1} E, \Lambda^{2} E, \ldots, \Lambda^{m-1} E \simeq E^{*}\right)$ is the unique minimal sequence of modules. In particular the dimension of $\mathbb{H}$ is at least $\sum_{k=1}^{m-1}\left(\begin{array}{c}m \\ k\end{array}\right)=2^{m}-2$.

Proof of Proposition 2.17. Write $\tilde{A}$ as

$$
\tilde{a}: E \otimes F^{*} \longrightarrow\left(\oplus_{i=1}^{m} \Lambda^{i} E \otimes \Lambda^{i} F^{*}\right) \otimes\left(\oplus_{j=0}^{m-1} \Lambda^{j} E \otimes \Lambda^{j} F^{*}\right)^{*} .
$$

For $u \in E \otimes F^{*}, \operatorname{Det}(\tilde{a}(u))$ belongs to

$$
\left(\Lambda^{n} \oplus_{j=0}^{m-1} \Lambda^{j} E \otimes \Lambda^{j} F^{*}\right)^{*} \otimes\left(\Lambda^{n} \oplus_{i=1}^{m} \Lambda^{i} E \otimes \Lambda^{i} F^{*}\right),
$$

which may be canonically identified with

$$
\left(\Lambda^{0} E \otimes \Lambda^{0} F^{*}\right)^{*} \otimes \Lambda^{m} E \otimes \Lambda^{m} F^{*} \simeq \Lambda^{m} E \otimes \Lambda^{m} F^{*}
$$

These identifications determine $\mathrm{GL}(E) \times \mathrm{GL}(F)$-equivariant polynomial maps

$$
E \otimes F^{*} \stackrel{\tilde{a}}{\longrightarrow}\left(\oplus_{i=1}^{m} \Lambda^{i} E \otimes \Lambda^{i} F^{*}\right) \otimes\left(\oplus_{j=0}^{m-1} \Lambda^{j} E \otimes \Lambda^{j} F^{*}\right)^{*} \stackrel{\mathcal{D} e t}{\longrightarrow} \Lambda^{m} E \otimes \Lambda^{m} F^{*}
$$

Choose bases $\mathcal{B}_{E}$ and $\mathcal{B}_{F}$, respectively of $E$ and $F$ to identify $E \otimes F^{*}$ with $\mathcal{M}_{m}(\mathbb{C})$. Choose a total order on the subsets of $\mathcal{B}_{E} \times \mathcal{B}_{F}$, to get bases of each $\Lambda^{j} E \otimes \Lambda^{j} F^{*}$ and maps

$$
\mathcal{M}_{m}(\mathbb{C}) \stackrel{\tilde{A}}{\longrightarrow} \mathcal{M}_{n}(\mathbb{C}) \stackrel{\operatorname{det}_{n}}{\longrightarrow} \mathbb{C} .
$$

As in the proof of Proposition 2.16, this implies that $\mathrm{GL}(E) \times \mathrm{GL}(F)$ belongs to the image of $\bar{\rho}_{A}$, so $P=\operatorname{det}_{n} \circ \tilde{A}$ is a scalar multiple of $\operatorname{det}_{m}$. 
To see it is the correct multiple, specialize to the diagonal matrices. Reorder the rows and columns so that all non-zero entries of $A\left(\mathcal{M}_{m}\left(\mathbb{C}_{m}\right)\right)$ appear in the upper-left corner. Note that since we made the same permutation to rows and columns this does not change the sign. Also note that since we have diagonal matrices, there are only plus signs for the entries of $A\left(\mathcal{M}_{m}\left(\mathbb{C}_{m}\right)\right)$. In fact this upper-left corner is exactly Grenet's representation for

$$
\operatorname{perm}_{m}\left(\begin{array}{ccc}
y_{1}^{1} & \cdots & y_{m}^{m} \\
& \vdots & \\
y_{1}^{1} & \cdots & y_{m}^{m}
\end{array}\right)
$$

which is $m !\left(y_{1}^{1} \cdots y_{m}^{m}\right)$. Finally note that each term in an expansion contains $n-m$ elements of $\Lambda_{0}$ to conclude.

It remains to prove that transp belongs to the image of $\bar{\rho}_{A}$. The following diagram is commutative:

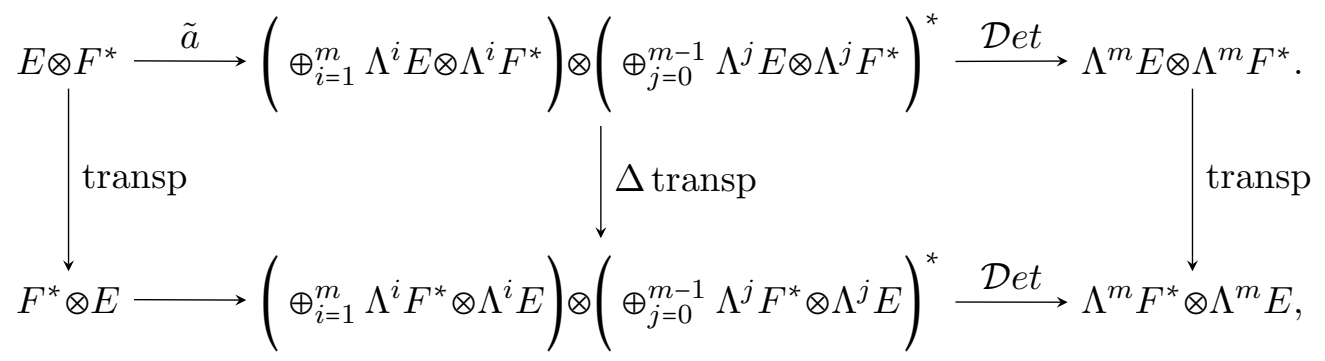

where $\Delta$ transp is the transposition on each summand.

Using $\mathcal{B}_{E}$ and $\mathcal{B}_{F}$, we identify the 6 spaces with matrix spaces. The first vertical map becomes the transposition from $\mathcal{M}_{m}(\mathbb{C})$ to itself. The last vertical map becomes the identity on $\mathbb{C}$. The middle vertical map is the endomorphism of $\mathcal{M}_{n}(\mathbb{C})$ corresponding to bijections between bases of spaces $\Lambda^{j} E \otimes \Lambda^{j} F^{*}$ and $\Lambda^{j} F^{*} \otimes \Lambda^{j} E$. It follows that there exist two permutation matrices $B_{1}, B_{2} \in \mathrm{GL}_{n}(\mathbb{C})$ such that

$$
\forall M \in \mathcal{M}_{m}(\mathbb{C}) \quad \tilde{A}\left(M^{T}\right)=B_{1} \tilde{A}(M) B_{2}{ }^{-1},
$$

proving that transp belongs to the image of $\bar{\rho}_{A}$.

\section{Proofs of Results on Determinantal RePresentations of$$
\operatorname{perm}_{m}
$$

Proofs of Propositions 2.9 and 2.10. The maps $s_{k}(v):\left(S^{k} E\right)_{\text {reg }} \rightarrow\left(S^{k+1} E\right)_{\text {reg }}$ are related to the maps $e x_{k}(v): \Lambda^{k} E \rightarrow \Lambda^{k+1} E$ as follows. The sources of both maps have bases indexed by multi-indices $I=\left(i_{1}, \cdots, i_{k}\right)$ with $1 \leq i_{1}<$ $\cdots<i_{k} \leq m$, and similarly for the targets. The maps are the same on these basis vectors except for with $s_{k}(v)$ all the coefficients are positive whereas with $e x_{k}(v)$ there are signs. Thus the polynomial computed by (11) is the same as the polynomial computed by (14) except all the $y_{j}^{i}$ appear positively. Reviewing the sign calculation, we get the result. 
The maps $S_{k}$ and $E X_{k}$ are similarly related and we conclude this case similarly.

Remark 6.1. The above proof can be viewed more invariantly in terms of the Young-Howe duality functor described in [AW07].

Proof of Theorem 2.8. Write $E, F=\mathbb{C}^{m}$. Let $\tilde{A}$ be a determinantal representation of $\operatorname{perm}_{m}$ such that $\tilde{A}(0)=\Lambda_{n-1}$. Embed $N\left(T^{\mathrm{GL}(E)}\right)$ in $\operatorname{GL}(\operatorname{Hom}(F, E))$ by $g \longmapsto\{M \mapsto g M\}$. We assume the image of $\bar{\rho}_{A}$ contains $N\left(T^{\mathrm{GL}(E)}\right)$. Set $N=N\left(T^{\mathrm{GL}(E)}\right)$ and $T=T^{\mathrm{GL}(E)}$.

As in the proof of Theorem 2.14, we get a reductive subgroup $L$ of $\left(\mathrm{GL}\left(\ell_{2}\right) \times \mathrm{GL}(\mathbb{H})\right) \rtimes \mathbb{Z}_{2}$ mapping onto $N$ by $\bar{\rho}_{A}$. In the determinant case, at this point we dealt with the universal cover of the connected reductive group $\mathrm{GL}(E)$. Here the situation is more complicated for two reasons. First, there is no "finite universal cover" of $\mathfrak{S}_{m}$ (see e.g. [Ste89, Józ89]). Second, since our group is not connected, we will have to deal with the factor $\mathbb{Z}_{2}$ coming from transposition, which will force us to work with a subgroup of $N$. Fortunately this will be enough for our purposes.

We first deal with the $\mathbb{Z}_{2}$ : Since $L /\left(L \cap \mathbb{G}_{\operatorname{det}_{n}, \Lambda_{n-1}}^{\circ}\right)$ embeds in $\mathbb{G}_{\operatorname{det}_{n}, \Lambda_{n-1}} / \mathbb{G}_{\operatorname{det}_{n}, \Lambda_{n-1}}^{\circ} \simeq$ $\mathbb{Z}_{2}$, the subgroup $L \cap \mathbb{G}_{\operatorname{det}_{n}, \Lambda_{n-1}}^{\circ}$ has index 1 or 2 in $L$. Since the alternating group $\mathfrak{A}_{m}$ is the only index 2 subgroup of $\mathfrak{S}_{m}, \bar{\rho}_{A}\left(L \cap \mathbb{G}_{\operatorname{det}_{n}, \Lambda_{n-1}}^{\circ}\right)$ contains $T \rtimes \mathfrak{A}_{m} \subset N$. In any case, there exists a reductive subgroup $L^{\prime}$ of $L$ such that $\bar{\rho}_{A}\left(L^{\prime}\right)=T \rtimes \mathfrak{A}_{m} \subset N$.

Next we deal with the lack of a lift. We will get around this by showing we may label irreducible $L^{\prime}$ modules only using labels from $\bar{\rho}_{A}\left(L^{\prime}\right)=T^{\mathrm{GL}(E)} \rtimes$ $\mathfrak{A}_{m}$.

The connected reductive group $L^{\prime \circ}$ maps onto $T$. Let $Z$ denote the center of $L^{\prime \circ}$; then $\bar{\rho}_{A}\left(Z^{\circ}\right)=T$. In particular the character group $X(T)$ may be identified with a subgroup of the character group $X\left(Z^{\circ}\right)$. The action of $L^{\prime}$ by conjugation on itself induces an action of the finite group $L^{\prime} / L^{\prime \circ}$ on $Z^{\circ}$. Moreover, the morphism $\bar{\rho}_{A}$ induces a surjective map $\pi_{A}: L^{\prime} / L^{\prime \circ} \longrightarrow \mathfrak{A}_{m}$. These actions are compatible in the sense that for $g \in L^{\prime} / L^{\prime \circ}, t \in T, z \in Z^{\circ}$ and $\sigma \in \mathfrak{A}_{m}$ satisfying $\pi_{A}(g)=\sigma$ and $\bar{\rho}_{A}(z)=t$,

$$
\sigma \cdot t=\bar{\rho}_{A}(g \cdot z) .
$$

In particular, both the kernel of $\bar{\rho}_{A}$ restricted to $Z^{\circ}$ and $X(T)$ are stable under the $\left(L^{\prime} / L^{\prime \circ}\right)$-action.

Let $\Gamma_{\mathbb{Q}}$ be a complement of the subspace $X(T) \otimes \mathbb{Q}$ in the vector space $X\left(Z^{\circ}\right) \otimes \mathbb{Q}$ stable under the action of $\left(L^{\prime} / L^{\prime \circ}\right)$. Set $\Gamma=\Gamma_{\mathbb{Q}} \cap X\left(Z^{\circ}\right)$ and $\tilde{T}=\left\{t \in Z^{\circ}: \forall \chi \in \Gamma \quad \chi(t)=1\right\}$. Then $\tilde{T}$ is a subtorus of $Z^{\circ}$ and the restriction of $\bar{\rho}_{A}$ to $\tilde{T}$ is a finite morphism onto $T$.

The character group $X(\tilde{T})$ may be identified with $X\left(Z^{\circ}\right) / \Gamma$ by restriction. Then $X(T)$ may be identified with a subgroup of $X(\tilde{T})$ of finite index. Hence there exists a natural number $k_{0}$ such that $k_{0} X(\tilde{T}) \subset X(T) \subset X(\tilde{T})$.

Let $W$ be an irreducible representation of $L^{\prime}$. It decomposes under the action of $\tilde{T}$ as

$$
W=\oplus_{\chi \in X(\tilde{T})} W^{\chi}
$$


Set $\operatorname{Wt}(\tilde{T}, W)=\left\{\chi \in X(\tilde{T}): W^{\chi} \neq\{0\}\right\}$. The group $L^{\prime}$ acts by conjugation on $\tilde{T}$ and so on $X(\tilde{T})$. By the rigidity of tori (see e.g., [Hum75, §16.3]), $L^{\prime \circ}$ acts trivially. Hence, the finite group $L^{\prime} / L^{\prime \circ}$ acts on $\tilde{T}$ and $X(\tilde{T})$. For any $t \in \tilde{T}, h \in L^{\prime}$ and $v \in W, t h v=h\left(h^{-1} t h\right) v$. Hence $\operatorname{Wt}(\tilde{T}, W)$ is stable under the action of $L^{\prime} / L^{\prime \circ}$. For $\chi \in \mathrm{Wt}(\tilde{T}, W)$, the set $\oplus_{\sigma \in L^{\prime} / L^{\prime \circ}} W^{\sigma \cdot \chi}$ is stable under the action $L^{\prime}$. By irreducibility of $W$, one deduces that $\operatorname{Wt}(\tilde{T}, W)$ is a single $\left(L^{\prime} / L^{\prime \circ}\right)$-orbit. Then $k_{0} \operatorname{Wt}(\tilde{T}, W) \subset X(T)$ is an $\mathfrak{A}_{m}$-orbit.

We are now in a position to argue as in $\S 3.6$.

Let $\varepsilon_{i}$ denote the character of $T$ that maps an element of $T$ on its $\mathrm{i}^{\text {th }}$ diagonal entry. The set $\left\{a_{1} \varepsilon_{1}+\cdots+a_{m} \varepsilon_{m}: a_{1} \geq \cdots \geq a_{m-1}\right.$ and $\left.a_{m-2} \geq a_{m}\right\}$ is a fundamental domain of the action of $\mathfrak{A}_{m}$ on $X\left(T^{\mathrm{GL}(E)}\right)$. Such a weight is said to be $\mathfrak{A}_{m}$-dominant. Hence, there exists a unique $\mathfrak{A}_{m}$-dominant weight $\chi_{W}$ such that $k_{0} \mathrm{Wt}(\tilde{T}, W)=\mathfrak{A}_{m} \cdot \chi_{W}$.

Summary of the properties of $L^{\prime}, \tilde{T}$ and $k_{0}$ :

(1) $L^{\prime}$ is a reductive subgroup of $\operatorname{GL}\left(\ell_{2}\right) \times \mathrm{GL}(\mathbb{H})$;

(2) $\bar{\rho}_{A}\left(L^{\prime}\right)=\mathfrak{A}_{m} \ltimes T$;

(3) $\tilde{T}$ is a central subtorus of $\left(L^{\prime}\right)^{\circ}$;

(4) $\bar{\rho}_{A}: \tilde{T} \longrightarrow T$ is finite and surjective, inducing embeddings $k_{0} X(\tilde{T}) \subset$ $X(T) \subset X(\tilde{T})$

(5) For any irreducible representation $W$ of $L^{\prime}$ there exists a unique $\mathfrak{A}_{m}$-dominant weight $\chi_{W}$ such that $k_{0} \mathrm{Wt}(\tilde{T}, W)=\mathfrak{A}_{m} \cdot \chi_{W}$.

(6) For the standard representation $E$ of $L^{\prime}$ through $\bar{\rho}_{A}, \chi_{E}=k_{0} \varepsilon_{1}$.

The action of $L^{\prime}$ on $\mathcal{M}_{n}(\mathbb{C})=\left(\ell_{1} \oplus \mathbb{H}\right)^{*} \otimes\left(\ell_{2} \oplus \mathbb{H}\right)$ respects the decomposition

$$
\mathcal{M}_{n}(\mathbb{C})=\ell_{1}^{*} \otimes \ell_{2} \oplus \ell_{1}^{*} \otimes \mathbb{H} \oplus \mathbb{H}^{*} \otimes \ell_{2} \oplus \mathbb{H}^{*} \otimes \mathbb{H} .
$$

The image of $A$ is an $L^{\prime}$-module isomorphic to the sum of $m$ copies of $E$. In particular, its projection on $\ell_{1}^{*} \otimes \ell_{2}$ has to be zero. Hence

$$
A(V) \subset \ell_{1}^{*} \otimes \mathbb{H} \oplus \mathbb{H}^{*} \otimes \ell_{2} \oplus \mathbb{H}^{*} \otimes \mathbb{H} .
$$

As was the case before, for the determinant to be non-zero, we need the projection to $\ell_{1}^{*} \otimes \mathbb{H}$ to be non-zero, so it must contain at least one copy $\mathbb{H}_{1}$ of $E$.

Assume first that $\mathbb{H}_{1}^{*} \otimes \ell_{2} \simeq E$. This happens only if $m=2$, where perm $m$ is a quadric.

Assume now that $\mathbb{H}_{1}^{*} \otimes \ell_{2} \neq E$. Choose an $L^{\prime}$-stable complement $\mathbb{S}_{1}$ of $\mathbb{H}_{1}$ in $\mathbb{H}$. If the projection of $A(V)$ on $\mathbb{H}_{1}^{*} \otimes \mathbb{S}_{1}$ is zero, one can discard $\mathbb{H}_{1}$ and start over as in the proof of the determinant case, so we assume it contributes non-trivially. Continuing so on, one gets a sequence $\mathbb{H}_{1}, \ldots, \mathbb{H}_{k}$ of irreducible $L^{\prime}$-submodules of $\mathbb{H}$ in direct sum such that

(1) $k \geq 2$;

(2) $E \subset \mathbb{H}_{i}^{*} \otimes \mathbb{H}_{i+1}$ for any $i=1, \ldots, k-1$;

(3) $E \simeq \mathbb{H}_{k}^{*} \otimes \ell_{2}$.

Let $\gamma: \mathbb{C}^{*} \longrightarrow \tilde{T}$ be a group homomorphism such that $\bar{\rho}_{A} \circ \gamma(t)=t^{k_{0}} \operatorname{Id}_{E}$. Then $\gamma\left(\mathbb{C}^{*}\right)$ acts trivially on $\mathbb{H}_{1}^{*} \otimes \mathbb{H}_{1}$ and with weight $k_{0}$ on $E$. Hence the projection of $A(V)$ on $\mathbb{H}_{1}^{*} \otimes \mathbb{H}_{1}$ is zero. (Recall that via $\Lambda, \operatorname{Id}_{\mathbb{H}_{1}} \in \mathbb{H}_{1}^{*} \otimes \mathbb{H}_{1}$.) 
More generally the action of $\gamma$ shows that the non-zero blocks of $A(V)$ are $\ell_{1}^{*} \otimes \mathbb{H}_{1}, \mathbb{H}_{i}^{*} \otimes \mathbb{H}_{i+1}$, and $\mathbb{H}_{k}^{*} \otimes \ell_{2}$. Consider the following picture:

$$
\left(\begin{array}{ccc}
0 & \mathbb{H}_{1}^{*} \otimes \ell_{2} & \cdots \\
E & \mathrm{Id}_{\mathbb{H}_{1}} & \cdots \\
\vdots & \mathbb{H}_{1}^{*} \otimes S_{1} & \cdots
\end{array}\right)
$$

Write

$$
k_{0} \mathrm{Wt}\left(\tilde{T}, \mathbb{H}_{i}\right)=\mathfrak{A}_{m} \cdot \chi_{\mathbb{H}_{i}}
$$

Then

$$
k_{0} \mathrm{Wt}\left(\tilde{T}, \mathbb{H}_{i}^{*} \otimes \mathbb{H}_{i+1}\right)=\left\{-\sigma_{1} \chi_{\mathbb{H}_{i}}+\sigma_{2} \chi_{\mathbb{H}_{i+1}}: \sigma_{1}, \sigma_{2} \in \mathfrak{A}_{m}\right\} .
$$

This set has to contain $k_{0} \mathrm{Wt}(\tilde{T}, E)=\left\{k_{0} \varepsilon_{i} \mid i \in[m]\right\}$. We deduce that $\chi_{\mathbb{H}_{i+1}}=\sigma \chi_{\mathbb{H}_{i}}+k_{0} \varepsilon_{u}$ for some $\sigma \in \mathfrak{A}_{m}$ and $u \in[m]$.

We define the length $\ell(\chi)$ of $\chi \in X(T)$ as its number of non-zero coordinates in the basis $\left(\varepsilon_{1}, \ldots, \varepsilon_{m}\right)$. Then

$$
\ell\left(\chi_{\mathbb{H}_{i+1}}\right) \leq \ell\left(\chi_{\mathbb{H}_{i}}\right)+1 \text {. }
$$

Observe that $\chi_{\ell_{2}}$ is invariant under $\mathfrak{A}_{m}$. Hence $\chi_{\ell_{2}}=\alpha\left(\varepsilon_{1}+\cdots+\varepsilon_{m}\right)$ for some $\alpha \in \mathbb{Z}$. The action of $\gamma$ shows that $\alpha=k-1$. We deduce that $\ell\left(\chi_{\mathbb{H}_{k}}\right) \geq m-1$. Then, by inequality (19), there exists a subset $\mathbb{H}_{i_{1}}, \ldots, \mathbb{H}_{i_{m-1}}$ of the $\mathbb{H}_{j}$ 's with $\ell\left(\chi_{\mathbb{H}_{i s}}\right)=s$.

We claim that $\operatorname{dim}\left(\mathbb{H}_{i_{s}}\right) \geq\left(\begin{array}{c}m \\ s\end{array}\right)$. First, $\operatorname{dim}\left(\mathbb{H}_{i_{s}}\right)$ is greater or equal to the cardinality of $\mathfrak{A}_{m} \chi_{\mathbb{H}_{i}}$. Since $m \geq 3, \mathfrak{A}_{m}$ acts transitively on the subsets of $[m]$ with $s$ elements. The claim follows.

Summing these inequalities on the dimension of the $\mathbb{H}_{i_{s}}$, we get

$$
\operatorname{dim} \mathbb{H} \geq \sum_{j=0}^{m-1} \operatorname{dim} \mathbb{H}_{i_{j}} \geq \sum_{j=1}^{m-1}\left(\begin{array}{c}
m \\
j
\end{array}\right)=2^{m}-2 .
$$

Proof of Theorem 2.1. This proof is omitted since it is very similar to the proof of Theorem 2.13.

\section{REFERENCES}

[ABV15] J. Alper, T. Bogart, and M. Velasco, A lower bound for the determinantal complexity of a hypersurface, ArXiv e-prints (2015).

[AW07] Kaan Akin and Jerzy Weyman, Primary ideals associated to the linear strands of Lascoux's resolution and syzygies of the corresponding irreducible representations of the Lie superalgebra $\mathbf{g l}(m \mid n)$, J. Algebra 310 (2007), no. 2, 461-490.

[BBR94] David A. Mix Barrington, Richard Beigel, and Steven Rudich, Representing Boolean functions as polynomials modulo composite numbers, Comput. Complexity 4 (1994), no. 4, 367-382, Special issue on circuit complexity (Barbados, 1992). MR 1313536 (96b:68058)

[Cai90] Jin-Yi Cai, A note on the determinant and permanent problem, Inform. and Comput. 84 (1990), no. 1, 119-127.

[CCL10] Jin yi Cai, Xi Chen, and Dong Li, Quadratic lower bound for permanent vs. determinant in any characteristic., Computational Complexity 19 (2010), no. 1, $37-56$.

[Com02] P. Comon, Tensor decompositions, state of the art and applications, Mathematics in Signal Processing V (J. G. McWhirter and I. K. Proudler, eds.), Clarendon Press, Oxford, UK, 2002, arXiv:0905.0454v1, pp. 1-24. 
[ELSW15] K. Efremenko, J. M. Landsberg, H. Schenck, and J. Weyman, On minimal free resolutions and the method of shifted partial derivatives in complexity theory, ArXiv e-prints (2015).

[Fis94] Ismor Fischer, Sums of Like Powers of Multivariate Linear Forms, Math. Mag. 67 (1994), no. 1, 59-61.

[Fro97] G. Frobenius, Über die Darstellung der endlichen Gruppen durch lineare Substitutionen, Sitzungsber Deutsch. Akad. Wiss. Berlin (1897), 994-1015.

[Gly10] David G. Glynn, The permanent of a square matrix, European J. Combin. 31 (2010), no. 7, 1887-1891.

[Gre14] Bruno Grenet, An Upper Bound for the Permanent versus Determinant Problem, Theory of Computing (2014), Accepted.

[Hum75] James E. Humphreys, Linear algebraic groups, Springer-Verlag, New YorkHeidelberg, 1975, Graduate Texts in Mathematics, No. 21.

[Józ89] Tadeusz Józefiak, Characters of projective representations of symmetric groups, Exposition. Math. 7 (1989), no. 3, 193-247.

[Lan] J.M. Landsberg, Geometry and complexity theory, preprint available at http://www.math.tamu.edu/ jml/simonsclass.pdf.

[MM61] Marvin Marcus and Henryk Minc, On the relation between the determinant and the permanent, Illinois J. Math. 5 (1961), 376-381.

[MR04] Thierry Mignon and Nicolas Ressayre, A quadratic bound for the determinant and permanent problem, Int. Math. Res. Not. (2004), no. 79, 4241-4253.

[MS01] Ketan D. Mulmuley and Milind Sohoni, Geometric complexity theory. I. An approach to the P vs. NP and related problems, SIAM J. Comput. 31 (2001), no. 2, 496-526 (electronic).

[MS08] - Geometric complexity theory. II. Towards explicit obstructions for embeddings among class varieties, SIAM J. Comput. 38 (2008), no. 3, 1175-1206.

[Mul] Ketan D. Mulmuley, On P vs NP, geometric complexity theory, and the flip I: a highlevel view, Technical Report TR200709, Computer Science Department, The University of Chicago, july 2007. Also available as arXiv:0709.0748.

[OV90] A. L. Onishchik and È. B. Vinberg, Lie groups and algebraic groups, Springer Series in Soviet Mathematics, Springer-Verlag, Berlin, 1990, Translated from the Russian and with a preface by D. A. Leites.

[RS11] Kristian Ranestad and Frank-Olaf Schreyer, On the rank of a symmetric form, J. Algebra 346 (2011), 340-342.

[Ste89] John R. Stembridge, Shifted tableaux and the projective representations of symmetric groups, Adv. Math. 74 (1989), no. 1, 87-134.

[Val79] Leslie G. Valiant, Completeness classes in algebra, Proc. 11th ACM STOC, 1979, pp. 249-261.

[Val84] L. G. Valiant, Short monotone formulae for the majority function, J. Algorithms 5 (1984), no. 3, 363-366. MR 756162 (86d:94041)

[vzG87] Joachim von zur Gathen, Permanent and determinant, Linear Algebra Appl. 96 (1987), 87-100.

[Yab15] Akihiro Yabe, Bi-polynomial rank and determinantal complexity, CoRR abs/1504.00151 (2015).

E-mail address: jml@math.tamu.edu, ressayre@math.univ-lyon1.fr 